\title{
Precision ring rolling technique and application in high-performance bearing manufacturing
}

\author{
Lin Hua ${ }^{1,3, a}$, Jiadong Deng ${ }^{1,3}$, and Dongsheng Qian ${ }^{2,3}$ \\ ${ }^{1}$ School of Automotive Engineering, Wuhan University of Technology, 430070 Wuhan, China \\ ${ }^{2}$ School of Materials Science and Engineering, Wuhan University of Technology, \\ 430070 Wuhan, China \\ ${ }^{3}$ Hubei Key Laboratory of Advanced Technology for Automotive Components, 430070 Wuhan, China
}

\begin{abstract}
High-performance bearing has significant application in many important industry fields, like automobile, precision machine tool, wind power, etc. Precision ring rolling is an advanced rotary forming technique to manufacture high-performance seamless bearing ring thus can improve the working life of bearing. In this paper, three kinds of precision ring rolling techniques adapt to different dimensional ranges of bearings are introduced, which are cold ring rolling for small-scale bearing, hot radial ring rolling for medium-scale bearing and hot radial-axial ring rolling for large-scale bearing. The forming principles, technological features and forming equipments for three kinds of precision ring rolling techniques are summarized, the technological development and industrial application in China are introduced, and the main technological development trend is described.
\end{abstract}

\section{Introduction}

Bearings are the key basic components of mechanical equipment for bearing load and transmitting power. They are widely used in the fields of machine tool, automobile, railway, ship, energy, aerospace, etc. Bearings which are used in precision numerical control (NC) machine tool, high-speed railway, wind power, aircraft engine, etc. are not only owning precise size, but also working with long life and high reliability in the strict conditions of high speed, heavy load, high temperature and impact load. These bearings belong to high-performance bearings. They represent bearings' performance level and reflect national manufacturing level.

A bearing is usually composed by bearing rings, bearing rollers and bearing cage. Bearing rings are basic body of a bearing. The weight of bearing rings accounts for $60 \sim 70 \%$ of the total weight, and the cost accounts for $60 \sim 70 \%$ of the total cost. Therefore, bearing rings determine the precision and performance of a bearing. The traditional manufacturing method is forging to obtain billet and cutting to obtain final ring, which has disadvantages of high energy cost, low material utilization,

\footnotetext{
${ }^{a}$ Corresponding author: hualin@whut.edu.cn
}

This is an Open Access article distributed under the terms of the Creative Commons Attribution License 4.0, which permits unrestricted use, distribution, and reproduction in any medium, provided the original work is properly cited. 
a)

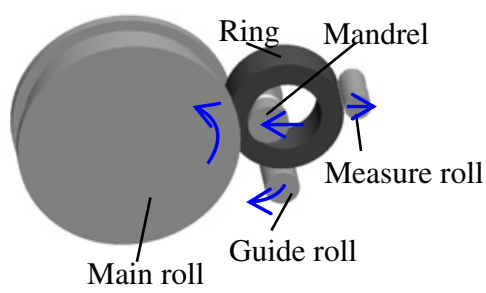

c)

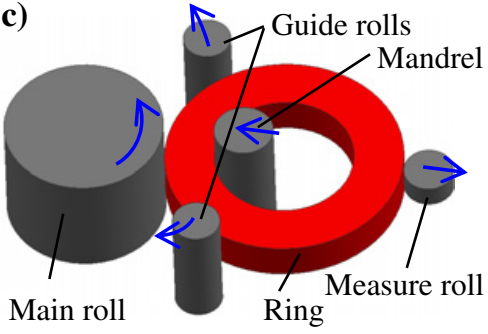

b)
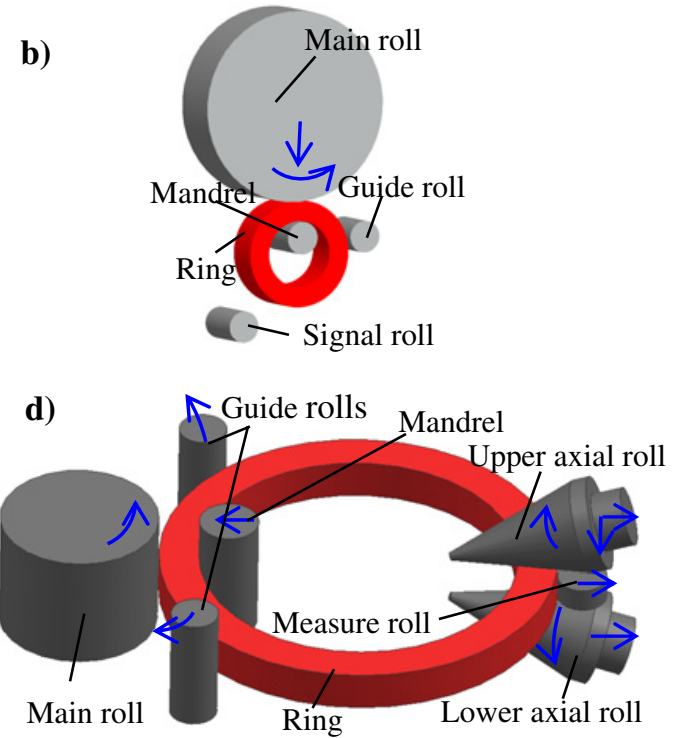

Figure 1. Forming principle of ring rolling: a) CRR; b) vertical HRRR; c) horizontal HRRR; d) HRARR.

low productivity, coarse and uneven microstructures, incomplete metal streamlines, poor mechanical properties. It is difficult to meet the usage requirement of high-performance bearing.

Ring rolling is a precise rotary forming technique to manufacture seamless rings, which makes rings producing local plastic deformation continuously to reduce wall-thickness, enlarge diameter and shape profile by rotation driving and linear feeding of the rolls $[1,2]$. In this way, the required geometry size and microstructure property are obtained. Bearing rings formed by ring rolling are not only saving energy and material cost, but also improving production efficiency, mechanical performance and working life [3]. The microstructure is dense and the metal streamlines distribute along raceway. Thus, ring rolling has been widely applied in bearing ring manufacturing and become an indispensable and advanced forming technology to manufacture high-performance bearings.

\section{Typical ring rolling technologies for bearing ring}

\subsection{Cold ring rolling for small-scale bearing ring}

Cold ring rolling (CRR) is an advanced rotary forming technique to manufacture seamless ring under room temperature by CRR mill [1]. With continuous local extrusion in radial rolling pass, ring radial thickness decrease, ring diameter enlarge, and cross-section shape. CRR mill main includes main roll part, mandrel part, guide part, measure part, frame part, power and control system. During rolling process, main roll makes active rotation, mandrel makes linear feed, guide roll moves according to ring's expanding, measure roll measures ring's diameter. When the ring's diameter enlarge to the target value, the rolling process finishes. This technique is suited to produce small-scale bearing ring whose diameter is smaller than $250 \mathrm{~mm}$.

\subsection{Hot radial ring rolling for medium-scale bearing ring}

Hot radial ring rolling (HRRR) is an advanced rotary forming technique to manufacture seamless ring under high temperature by HRRR mill [1]. Similar to CRR, with continuous local extrusion in radial 
rolling pass, ring radial thickness decrease, the ring diameter enlarge, and the cross-section shape. According to the relationship between direction of roll's axle and horizontal direction, HRRR mill can be divided to vertical HRRR mill and horizontal HRRR mill. The direction of roll's axle in vertical HRRR mill is parallel to horizontal direction. During rolling process, main roll makes active rotation and linear feed, mandrel makes passive rotation, guide roll is fixed in the exit side of main roll, signal roll locates at the opposite side of the guide roll. When the ring's diameter enlarge to contact the guide roll, the rolling process finishes. The direction of roll's axle in horizontal HRRR mill is vertical to horizontal direction. During rolling process, main roll makes active rotation, mandrel makes linear feed, two guide rolls locate symmetrically at the two sides of main roll and moves according to ring's expanding, signal roll locates at the opposite side of the main roll to measure ring' diameter. When the ring's diameter enlarge to the target value, the rolling process finishes. This technique is suited to produce mediumscale bearing ring whose diameter ranges in $50 \mathrm{~mm}$ to $1000 \mathrm{~mm}$.

\subsection{Hot radial-axial ring rolling for large-scale bearing ring}

Hot radial-axial ring rolling (HRARR) is an advanced rotary forming technique to manufacture seamless ring under high temperature by HRARR mill [1]. With continuous local extrusion in radial and axial rolling passes, ring radial thickness decrease, ring axial height decrease, ring diameter enlarge, and cross-section shape. Compared to horizontal HRRR mill, HRARR mill adds a pair of axial rolls to reduce ring's height. During rolling process, upper axial roll makes active rotation and linear feed downward, lower axial roll makes only active rotation. Two axial rolls are fixed on axial frame, which moves outward according to ring's expanding. This technique is suited to produce large-scale bearing ring whose diameter is larger than $1000 \mathrm{~mm}$.

\section{Development and application of precision ring rolling for bearing ring}

China is an important international market and manufacturing base of bearings. The market of bearings in china takes $10 \%$ of the total global market. Currently the annual output of bearings have reached nearly 20 billion sets, and the value is over $\$ 30$ billion a year. Facing the challenges from the globalization of manufacturing industry and the sustainable development, China is in the changing process from a big country into a powerful one in bearing manufacturing. With cooperation research of some bearing manufacturing enterprises, universities and research institutes, we aim at the autonomous development of precision ring rolling technology in high-performance bearing manufacturing, and finally get great technical innovations and breakthrough in precision CRR, HRRR and HRARR for bearing rings [3].

\subsection{Precision cold ring rolling for medium and small scale bearing ring}

\subsubsection{Precision cold ring rolling technology}

Based on the international semi-precision CRR technology, we innovated and developed a precision CRR technology for bearing ring manufacturing. The main technological process is as follows: cutting bars $\rightarrow$ making blanks by hot forging $\rightarrow$ preprocessing $\rightarrow$ rough turning $\rightarrow$ precision CRR $\rightarrow$ quenching $\rightarrow$ grinding. Through controlling blank precision, we improve CRR precision, and realize near net shape forming. The main technical advantages are as follows: 1) Improving CRR precision. The geometric precision level has reached $7 \sim 8$ grades and the surface finish has reached Ra $0.8 \sim 1.6 \mu \mathrm{m}$, which is higher than semi-precision CRR $1 \sim 2$ grades. 2) Improving material utilization ratio and production efficiency. The bearing ring raceway can be formed without turning processing, which reduces materials cost and labor hours consumed in turning processing. 3) Improving ring's mechanical 
a)

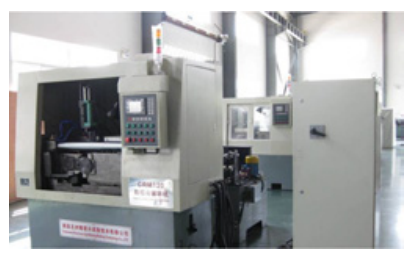

b)

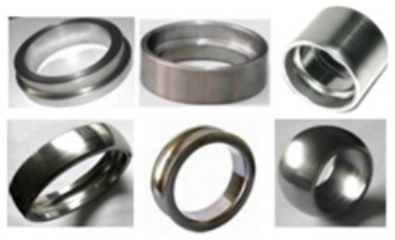

Figure 2. a) A new electromechanical servo NC precision CRR mill; b) machine tool and automobile bearing rings formed by precision CRR.

properties. The raceways formed by precision CRR without turning remain dense microstructure and complete metal streamline, thus it can reduce the damage to mechanical properties in turning process.

\subsubsection{Precision cold ring rolling equipment}

A new NC precision CRR mill was developed independently. And a servo motor-reducer-strong ball screw machine was creatively designed and developed, which replaced hydraulic servo system and acted as the main force transfer-feed system of CRR mill. The precise feed under a heavy load of 50 tons was realized. Its feed accuracy reaches $0.005 \mathrm{~mm}$, and the machining accuracy $\Phi 100 \pm 0.02 \mathrm{~mm}$, which is higher than the same type precision CRR mill of German and Japan, whose accuracy is $\Phi 100 \pm 0.1 \mathrm{~mm}$. The new type of CRR mill made a breakthrough to the limitation of traditional CRR mill using hydraulic cylinder as the main force transfer-feed system. It ensured the accuracy of precision CRR through improving equipment structure, which realized the transformation of ring rolling mill's structure and design method.

With application of precision CRR technology and equipment, some cold rolling products including ball bearing rings, roller bearing rings, joint bearing rings were developed and produced in a mass. And they were used in high-performance machine tool and automobile bearing. Bearing rings formed by precision CRR is widely exported to SKF, FAG, NSK, TIMKEN and other international famous bearing companies.

\subsubsection{Key technology of precision cold ring rolling}

The key technologies of high-performance bearing ring by precision CRR are as follows: 1) High plasticity pretreatment of bearing steel through CRR deformation at room temperature; 2) Design and manufacture of roll pass with long life; 3) Process design of precision CRR; 4) On-line precision measurement and control in CRR process.

\subsection{Precision hot radial ring rolling for large-scale profile bearing ring}

\subsubsection{Precision hot radial ring rolling technology}

The traditional hot ring rolling technology of large complex conical roller bearing rings which are used for high-speed railway locomotives and overloaded railway wagons is usually formed with a simplified cross-section firstly, then cut out the perfectly cross-section. The technical and economic effect is poor. Therefore, we exploited a precision HRRR technology for this kind of bearing rings. The main technological process is as follows: cutting bars $\rightarrow$ making blanks by hot extrusion forming $\rightarrow$ precision hot ring rolling $\rightarrow$ cutting $\rightarrow$ heat treatments $\rightarrow$ grinding. The new process forms out the complex cross-section of bearing rings directly by improving size precision of blank and process control of HRRR. Thus it saves a lot of cutting consumption and improves the performance of product. 
a)

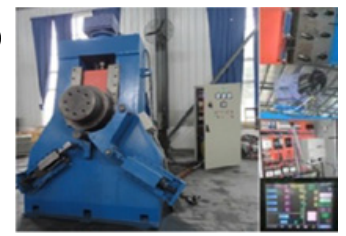

b)

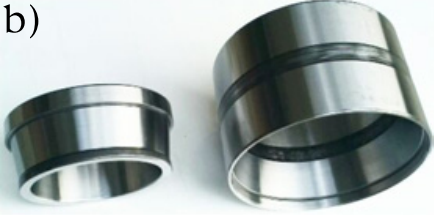

Figure 3. a) A new electromechanical servo NC precision HRRR mill; b) high-speed rail bearing rings formed by precision HRRR.

Compared to traditional HRRR, the improved forming process can save materials by $15-20 \%$, improve productivity by 3 times, and reduce production costs by $20 \%$.

\subsubsection{Precision hot radial ring rolling equipment}

Based on the research of NC precision CRR mill, we developed a NC precision HRRR mill. Similarly, hydraulic servo system was replaced by electromechanical servo system as the main force transferfeed system. By this way, the feeding accuracy reaches $0.01 \mathrm{~mm}$, and the forming accuracy reaches $\Phi 100 \pm 0.25 \mathrm{~mm}$. The automatic measurement control of the improved precision NC rolling mill replaces the manual control of the traditional half $\mathrm{NC}$ rolling mill. Thus it ensures the dimensional accuracy and stability, and improves the automation degree of the hot ring rolling process.

With application of precision radial ring rolling technology and equipment, we have developed many products, such as tapered roller bearings, cylindrical roller bearings, ball bearing, etc. And the products can be manufactured quantity, which are widely used in high-performance bearings of highspeed railway machine tool and overloaded metallurgical rolling mill.

\subsubsection{Key technology of precision hot radial ring rolling}

The key technologies of high performance bearing ring by precision HRRR are as follows: 1) Matching design between precision making blank and HRRR process; 2) Precision cooling control during hot rolling; 3) On-line precision measurement and control during HRRR.

\subsection{Precision hot radial-axial ring rolling for super large scale bearing ring}

\subsubsection{Precision hot radial-axial ring rolling technology}

The traditional manufacturing process of super large-scale wind power bearing whose diameter is more than one meter is as follows: cutting bars $\rightarrow$ heating bars $\rightarrow$ making blanks by hot freedom forging $\rightarrow$ mandrel reaming process $\rightarrow$ machining. Mandrel reaming process requires repeated heating and consumes lots of energy, materials and time, but it cannot form out raceway perfectly, which cause large subsequent machining capacity and poor product performance. Therefore, precision HRARR for super large scale bearing was developed. It mainly includes: cutting bars $\rightarrow$ heating bars $\rightarrow$ making blanks by hot freedom forging $\rightarrow$ mandrel reaming process $\rightarrow$ machining. Replacing mandrel reaming process by precision HRARR, can achieve large scale bearing ring rolling with heating one time and form out raceway directly, save energy for 30-40\%, save material for $25-30 \%$, increase productivity more than five times, reduce production costs by $30 \%$. Besides, the technology breaks through the bearing ring size limit of mandrel reaming process, achieves super lager scale bearing ring forming whose diameter is more than 5 meters. 
a)

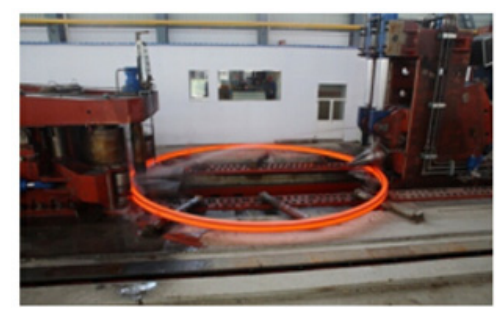

b)

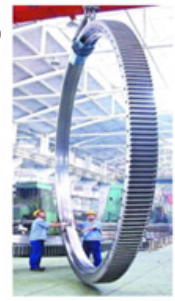

Figure 4. a) A super large scale HRARR mill RAM 9000; b) a super large scale wind power bearing ring formed by precision HRARR.

\subsubsection{Precision hot radial-axial ring rolling equipment}

We developed a super large scale NC precision HRARR mill. The largest diameter of rolled ring can reach 10 meters, and the largest height of rolled ring can reach 1.7 meters. The radial rolling force is 800 tons, and the axial is 600 tons, which ensures to rolling bearing rings with super lager scale.

Application of precision HRARR technology and equipment, 1 meter ring or larger ones such as ball bearings, wheel bearings, etc. were developed and achieved mass production. The technology and equipment are also widely used in high-performance wind power bearing. With application of precision HRARR technology and equipment, we have developed many products whose diameter larger than 1 meter, such as ball bearings, wheel bearings, etc. And the products can be manufactured quantity, which are widely used in high-performance bearings of wind power. These bearing rings are exported to America GE, Denmark VESTAS and other international famous wind power companies.

\subsubsection{Key technology of precision hot radial-axial ring rolling}

The key technologies of high performance bearing ring by precision HRARR are as follows: 1) Design of deformation ratio between radial direction and axial direction; 2) Design of process plan and transitory stage; 3) Stability control of HRARR process.

\section{Development trend of precision ring rolling technology for bearing ring}

As a necessary development requirement of high-level major equipment, high-performance bearing is the development direction of bearing. In order to match the development needs of high-performance bearing product, technology and market, Chinese precision ring rolling for bearing rings is developing towards the follow directions:

(1) Precision cold ring rolling for large scale bearing rings

Limited by properties of high carbon and chromium bearing steel GCr15, the size of bearing ring produced by CRR is smaller than $\Phi 250 \mathrm{~mm}$. In future, the forming technology and equipment of CRR for large-scale bearing rings whose diameter reach $\Phi 250 \mathrm{~mm}$ to $\Phi 500 \mathrm{~mm}$ need to be researched Besides, application of CRR technology for bearing ring should be expended.

(2) Control technology of hot ring rolling for bearing rings

At present, the technological conditions of bank making, ring rolling and cooling during hot rolling process for bearing rings fluctuate wildly, leading the geometric dimension and microstructure property of hot-rolled bearing rings unstable. In future, the control technology of hot rolling for bearing ring need to be researched. Controlling heating condition before rolling, 
rolling deformation during rolling and cooling condition after rolling is a possible approach to achieve precision control of geometric accuracy and microstructure property during hot rolling process of bearing rings, to ensure the consistency for geometric accuracy and microstructure property.

(3) Short process technology of ring rolling for bearing rings

At present, the blank for large scale bearing ring rolling need to be obtained by a series processes of smelting, casting steel ingots, cutting ingots, upsetting, punching, etc. Thus, the manufacturing process is so long. Many materials are wasted by upsetting and punching. Besides, the material was heated many times during the whole process, leading large energy consuming and material burning, which influences the blank quality. Therefore, a new process of casting and rolling compound technology was put forward. This method can significantly shorten the process by rolling the casting blank directly, which reduce blank making consumption and improve production efficiency. With the remarkable technical superiority, casting and rolling compound technology is expected to be an important development direction of bearing ring rolling technology in future.

\section{References}

[1] L. Hua, X.G. Huang, C.D. Zhu, Theory and Technology of Ring Rolling (China Machine Press, Beijing, 2001)

[2] Z.H. Hu, L. Hua, Technology of rotary metal forming (Chemical Industry Press, Beijing, 2010)

[3] L. Hua, D.S. Qian, Journal of Mechanical Engineering, 50, 70-76 (2014) 